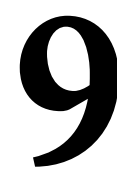

\title{
LA ATENCIÓN A LA DIVERSIDAD EN LOS MOOCS: UNA PROPUESTA METODOLÓGICA
}

\section{(ATTENTION TO DIVERSITY IN MOOCS: A METHODOLOGICAL PROPOSAL)}

\author{
Alba García Barrera \\ Universidad a Distancia de Madrid \\ Patricia Gómez Hernández \\ Carlos Monge López \\ Universidad de Alcalá \\ DOI: $10.5944 / e d u c X X 1.19038$
}

Cómo referenciar este artículo/How to reference this article:

García Barrera, A.; Gómez Hernández, P. y Monge López, C. (2017). La atención a la diversidad en los Moocs: Una propuesta metodológica. Educación XX1, 20(2), 215-233, doi: 10.5944/educXX1.19038

García Barrera, A.; Gómez Hernández, P. \& Monge López, C. (2017). La atención a la diversidad en los Moocs: Una propuesta metodológica. [Attention to diversity in Moocs: A methodological proposal]. Educación XX1, 20(2), 215-233, doi: 10.5944/educXX1.19038

\section{RESUMEN}

Los MOOCs se están convirtiendo en un recurso cada vez más frecuentemente utilizado a nivel mundial. Se ofertan desde todo tipo de entidades y ofrecen múltiples ventajas a nivel educativo, tales como ubicuidad, gratuidad, credibilidad y calidad. Sin embargo, las investigaciones acerca de sus aportaciones al conocimiento real de cada individuo apenas están comenzando. Los MOOCs aún son unos grandes desconocidos para la ciencia, y esta aún no puede garantizar que quienes certifican posean verdaderamente las habilidades que mediante ellos se acreditan. Además, cabe destacar que su principal virtud puede convertirse a su vez en su mayor inconveniente. La masificación de sus aulas virtuales implica que sus contenidos estén dirigidos a un público general y enfocados a un alumno de competencias bajas y medias. Sus creadores desconocen por completo las características que tienen los participantes, por lo que no pueden generar contenidos en función de sus conocimientos o experiencia previa. Su enorme alcance y su amplia gama de destinatarios hacen que adecuar la educación a las necesidades de cada estudiante se convierta en una compleja misión. Sin embargo, desde una revisión teórica, en el presente artículo se estudian las posibilidades que en realidad estos cursos ofrecen para personalizar la enseñanza y vencer los lastres que interpone la virtualidad en la educación a distancia. Se ofrecen algunas claves para poder adaptar los MOOCs sin olvidar atender a la diversidad siempre 
presente entre su alumnado. En consideraciones generales, pueden destacarse los aportes metodológicos y secuenciales para la implantación de un $M O O C$ desde una perspectiva de educación inclusiva).

\section{PALABRAS CLAVE}

Atención a la diversidad; $M O O C$; educación personalizada; diseño instruccional; autoaprendizaje; calidad educativa.

\section{ABSTRACT}

MOOCs are a resource that are becoming increasingly frequently used worldwide. They are offered from all types of institutions, especially universities, and they have many advantages for education such as ubiquity, gratuity, credibility and quality. However, research on their contributions to the actual knowledge of each individual are just beginning. MOOCs are still a big unknown for science, and it cannot be ensured that those who are certified truly possess the skills that are accredited through them. Also, note that its main virtue may become its biggest drawback. The overcrowding of virtual classrooms means that its contents are directed at the general public and targeted to a student of low and average skills. Its creators are completely unaware of the characteristics that participants have, and so cannot generate content based on their knowledge or experience. Its enormous scope and wide range of target makes adapting education to the needs of each student a complex task. However, from a theoretical review, this article discusses the potential that these courses really offer in order to personalize teaching and overcome the burdens imposed by virtuality in distance education. It also provides some keys to adapt MOOCs without forgetting to meet the ever-present diversity among its students. In general considerations, some methodological and sequential contributions can be highlighted for the implantation of a MOOC from an inclusive education perspective.

\section{KEYWORDS}

Attention to diversity; MOOC; personalized education; instructional design; self instruction; educational quality.

\section{INTRODUCCIÓN}

El origen de los MOOCs (Massive Open Online Courses) se suele asociar a los cursos ofertados por dos profesores universitarios de Stanford en el año 2011, pero en realidad parecen estar acuñados en asociación con un 
curso abierto en línea denominado «Connectivism and Connective Knowledge (CCK08)», que fue ofrecido en 2008 por la University of Manitoba en Canadá (Liyanagunawardena, Adams y Williams, 2013; Watters, 2013). Este curso parecía ser bastante diferente de las clases basadas en lecciones magistrales, de gestión del aprendizaje y/o de aquellos cursos ofertados por plataformas como Udacity, Coursera o $e d X$. Sus profesores procedían sobre todo del campo educativo y su preocupación consistía en construir redes y comunidades de aprendizaje en las que los estudiantes pensaran y participaran mediante el uso de tecnologías a su elección (blogs, vídeos de YouTube, debates síncronos, etc.). Dichas tecnologías eran agregadas a través de enlaces y feeds RSS al curso principal, que gozaba de libre acceso en la web (Watters, 2013).

Esta metodología parece encajar con una perspectiva conectivista del aprendizaje (Griesbaum, 2014; Levy, 2011). Dicha corriente subraya la importancia de las redes y conexiones de y para los recursos de aprendizaje, basándose en el valor que la diversidad de opiniones tiene en la adquisición y consolidación de conocimientos (Siemens, 2005) y relacionándose profundamente con las teorías de aprendizaje cooperativo (Kop y Hill, 2008). Tomando como punto de partida esta perspectiva, algunos denominan a estos cursos "MOOCs conectivistas» $\mathrm{O}$ «CMOOCs», interpretándolos como comunidades para la construcción conjunta de conocimiento que ayudan a incrementar tanto cuantitativa como cualitativamente el aprendizaje al considerarlo desde su vertiente más social. De este modo, los cMOOCs pretenden ir más allá del marco constructivista y convertirse en un modelo que interprete los aprendizajes horizontales que surgen en red (Siemens, 2006) y consiga así potenciarlos. En contraposición se encuentran los $X M O O C s$ o MOOCs masivos, otro tipo de MOOCs que rondan los 150.000 participantes (Downes, 2013, citado en Mackness, Waite, Roberts y Lovegrove, 2013), poseen un carácter eminentemente conductista y se configuran dentro de una metodología tradicional de enseñanza-aprendizaje (Méndez, 2013).

Dados los diferentes matices que se pueden encontrar en los distintos tipos de MOOCs, en este caso se destaca y apuesta por los de carácter conectivista, ya que presentan características que favorecen la interacción entre las personas y la adaptación del curso a las características de cada alumno que lo configura, fin que se persigue en este estudio.

Sin embargo, pese a la verdadera aportación al conocimiento que podría llegar a efectuarse desde un $C M O O C$ adecuadamente estructurado a nivel metodológico y organizativo, la realidad parece ser que en este tipo de cursos la participación de los usuarios no suele ser muy activa y además existe una considerable tasa de abandono. La causa parece resi- 
dir, por una parte, en las escasas posibilidades de interacción que existen con los expertos o profesores que tutorizan y/o dirigen el curso y, por otra, en la falta de socialización que se produce realmente en este entorno, así como en la brecha cultural y lingüística que a menudo se genera entre los participantes, especialmente si el curso es de carácter internacional. Todo ello origina en los estudiantes una sensación de soledad y desamparo que les provoca frustración cuando no saben cómo enfrentarse adecuadamente a la resolución de determinado problema, llegando a poner en duda sus propias capacidades (Calderón, Ezeiza y Jimeno, en prensa). Por ello, la figura del docente es crucial para que los alumnos se desenvuelvan correctamente y se sientan apoyados en todo el proceso de enseñanzaaprendizaje. Sin embargo, como ponen de manifiesto Martín-Monge, Bárcena y Read (2014) en un estudio de investigación-acción, no solamente es importante la interacción alumno-tutor, sino también alumno-alumno, con el fin de dar respuesta a una gran diversidad, especialmente lingüística, de los participantes.

Otro aspecto fundamental que hay que reseñar para comprender una de las principales problemáticas a las que se enfrentan los MOOCs se debe precisamente a su carácter masivo y abierto, que conlleva una inmensa diversidad del alumnado en cuanto a conocimientos previos sobre la materia, intereses, competencias tecnológicas, idioma, etc. (Méndez, 2013). Todo ello es un factor clave para el diseño y desarrollo de este tipo de cursos, que hasta el momento carece de una solución ampliamente aceptada. En este sentido, son escasas las investigaciones sobre la diversidad del alumnado participante en estos cursos, aunque bien es cierto que se comienzan a ver atisbos como el de Grünewald, Meinel, Totschnig y Willems (2013) sobre los diferentes estilos de aprendizaje.

No obstante, en el presente artículo se pretende ir un paso más allá, abordando la diversidad desde un punto de vista que atañe a las características que hacen de cada persona un ser humano diferente: intereses, motivaciones, competencias, capacidades, conocimientos, creencias, cultura, etc. Así, se defiende la idea de que en los MOOCs hay mucho más que compartir bajo el paraguas de la diversidad que una simple opinión o perspectiva, y no solo que compartir, sino cuidar y trabajar para que no afecte negativamente a los resultados de aprendizaje.

Por todo ello, el objetivo principal de este estudio es presentar una serie de propuestas metodológicas para incluir en la elaboración de los MOOCs a fin de atender lo máximo posible a la diversidad de su alumnado, sin olvidar las dificultades que habría que superar previamente en el momento de diseñarlos y que se exponen a continuación. 


\section{DIFICULTADES PARA ATENDER A LA DIVERSIDAD EN LOS MOOCS}

Pese a todas las ventajas que conllevan los MOOCs como entornos de aprendizaje, destacadas por múltiples autores a nivel internacional (Karsenti, 2013; Méndez, 2013; Odom, 2013), un examen más profundo y minucioso puede ofrecer otra perspectiva, la cual sugiere que dichos cursos no están cumpliendo del todo con las expectativas que generan y las promesas (y premisas) bajo las cuales surgieron. Desde este punto de vista resulta esencial examinar sus aportaciones en relación a los resultados y tipo de aprendizaje que producen, prestando especial atención al enfoque psicopedagógico que parecen estar tomando como base para su elaboración y diseño.

\section{ENTENDER LOS MOOCS DESDE LA PSICOPEDAGOGÍA}

La mayoría de los MOOCs parecen guiarse por un modelo tradicional de aprendizaje, basado en clases magistrales: se graban y publican unas cuantas sesiones cortas en forma de videoconferencia, de unos 5 o 20 minutos de duración, que quedan publicadas como lecciones para su posterior visualización por parte de los usuarios, quienes a continuación deberán responder una serie de cuestionarios autocorregibles sobre los aspectos abordados y, en el mejor de los casos, realizar tareas revisadas por pares. Dicha metodología, aunque cada vez más extendida, todavía cuenta con escasa evidencia científica que la respalde como forma eficaz de aprendizaje (Mackness y otros, 2013). De hecho, algunos autores están poniendo en tela de juicio dicho formato de enseñanza al considerar que realmente no resulta adecuado para algunas disciplinas académicas, áreas o materias, ni para aquellos estudiantes más acostumbrados a metodologías activas o experimentaciones prácticas (Watters, 2013).

Por todo ello, se hace necesario destacar que la masividad de los MOOCs genera una gran ventaja, que es que pueden albergar a una gran cantidad de interesados en el objeto de estudio que presenta cada curso, pero a la vez se convierte en un punto negativo y, por tanto, en una controversia de esta clase de cursos, ya que si no se da respuesta a las necesidades individuales y se atiende a las características particulares de cada alumno se pierde esta particularidad (la masividad) debido a que únicamente considera a los alumnos «modelo» (todos homogéneos y respondiendo a una media ficticia) a los que va destinado el curso.

En relación a las perversiones de la masificación, Valverde (2014) critica la estandarización del conocimiento, es decir, ofrecer los mismos contenidos y las mismas actividades para todos los estudiantes, acuñando el término «McDonalización» y señalando que el carácter masivo de los 
MOOCs es uno de sus elementos más emblemáticos y fue, inicialmente, una fortaleza para acabar en una de sus principales debilidades. Para las instituciones de Educación Superior, conseguir «audiencias» de centenares de miles de "alumnos» en todo el mundo a través de un curso online, supuso un sorprendente descubrimiento que fue erróneamente interpretado desde el punto de vista educativo. Probablemente las necesidades económicas de las universidades y su búsqueda de nuevas fuentes de financiación les hizo olvidar la incompatibilidad de lo «masivo» con la excelencia en la formación online. En una vuelta al siglo pasado, se abogó por la elaboración de materiales didácticos estandarizados, basados en la transmisión informativa y evaluados de manera automática a través de pruebas de elección múltiple. En estos diseños se desconsideran variables contextuales, culturales y de diferencias individuales (v.gr. conocimientos previos, estilos de aprendizaje). Se ofrecen como productos para un consumo inmediato, sencillo, rápido, sin elevados costes de producción, replicables sin limitación y con beneficios económicos directos. (p. 102).

Además, y poniendo un mayor énfasis en la masividad de estos cursos, que como ya se ha indicado es una de las características principales que todos los MOOCs comparten (Aguaded, 2013), cabe destacar que uno de sus objetivos o funciones consiste en enseñar masivamente determinada cuestión o conocimiento, centrándose más en los individuos como números que como personas. Por ello esta característica tal vez no sea la gran aportación de los MOOCs, sino que lo realmente interesante es que permiten manipular masas de datos en relación a los alumnos y, tras el análisis de estos, mejorar los procesos de enseñanza. Es decir, la forma de trabajo de estos cursos toma como base sistemas de minería de datos, sistemas inteligentes y análisis estadísticos (Köck, 2009) que recogen datos procedentes de las tareas de los estudiantes, tales como su perfil, su nivel de actividad, las decisiones que toman durante el curso, etc. (Calderón y otros, en prensa). Dicha información es accesible desde el sistema de administración que poseen los MOOCs, que recoge el análisis del aprendizaje de cada usuario y toma decisiones en consecuencia, generando un itinerario formativo particular para cada uno.

Toda esta recopilación de datos se podría justificar desde la perspectiva de la enseñanza adaptativa, que pretende personalizar el aprendizaje de cada estudiante partiendo de este análisis de datos, de modo que en función de las características de su propio proceso de estudio se facilite un andamiaje adecuado para la adquisición y construcción de sus nuevos conocimientos. De esta forma se proporciona una respuesta específica a las necesidades de cada educando, ofreciéndole aquellos recursos, actividades y materiales que faciliten su aprendizaje. Como indican Torres-Díaz, Infante y Valdiviezo (2014), la masificación personalizada es ya posible en los MOOCs. 
No obstante, este proceso puede dar lugar a errores basados en la interpretación automática de estos datos por parte del sistema, ya que obvia el contexto y circunstancias personales que pueden estar influyendo en los resultados de aprendizaje del estudiante. De este modo, puede suceder que el sistema calcule un itinerario formativo personal para el alumno que realmente no se adapte a sus capacidades o conocimientos previos, debido a que en determinado momento su trayectoria pudo verse modificada en función de unos condicionantes externos no tomados en consideración para la realización de dicho cálculo. Además, este cálculo automático es criticado por determinados autores al considerar que responde a una perspectiva conductista del aprendizaje que considera al estudiante como un sujeto pasivo en la construcción de su propio aprendizaje (como Calderón y otros, en prensa). Por esta razón se considera de vital importancia la figura del tutor o asesor virtual en este tipo de cursos, quedando a disposición del alumnado en caso de que existan esta clase de circunstancias y ayudando, así, a reconducir su proceso de aprendizaje en base a una interpretación más real del mismo.

En este sentido y poniendo en el punto de mira la adecuación de los MOOCs a las características y necesidades individuales de los estudiantes, puede constatarse la escasez de estudios que abordan esta problemática en la literatura científica. No obstante, cabe decir que cada vez con más frecuencia están cobrando fuerza dentro de los MOOCs las comunidades de colaboración como medio para llegar al aprendizaje. De este modo se potencia la participación del alumnado y se da respuesta a algunas de las necesidades que este presenta, de manera que todos se nutren con las experiencias y conocimientos de los compañeros y construyen, cada uno de ellos, su propio conocimiento en función de sus necesidades de aprendizaje.

\section{LAS APORTACIONES DE LOS CMOOCS A LA ATENCIÓN A LA DIVERSIDAD}

Los $c$ MOOCs se basan, según Méndez (2013), en que la jerarquización que existe entre docente y alumnos se rompe, quedando estos al mismo nivel y construyendo, entre todos los implicados, comunidades de aprendizaje dentro de las cuales todos tienen algo que aportar al resto. Por tanto, el papel del docente dentro del proceso de enseñanza-aprendizaje cobra un papel importante y alejado del rol que tradicionalmente ha tenido asignado, y que se encontraba más bien limitado a acciones unidireccionales dirigidas a impartir una serie de conocimientos que al alumno le eran dados. Sin embargo, en los cMOOCs es el docente el encargado de ofrecer a sus estudiantes los contenidos de introducción a la temática y guiarlos en el resto del camino, siendo estos los encargados, según sus motivaciones e intereses, de construir su propio proceso de aprendizaje de forma activa y responsable. 
Continuando con esta misma idea, los principios de aprendizaje que deberían seguir y promover los $c M O O C$ s serían fundamentalmente cuatro (Downes, 2007): autonomía, diversidad, apertura e interactividad (Figura 1).

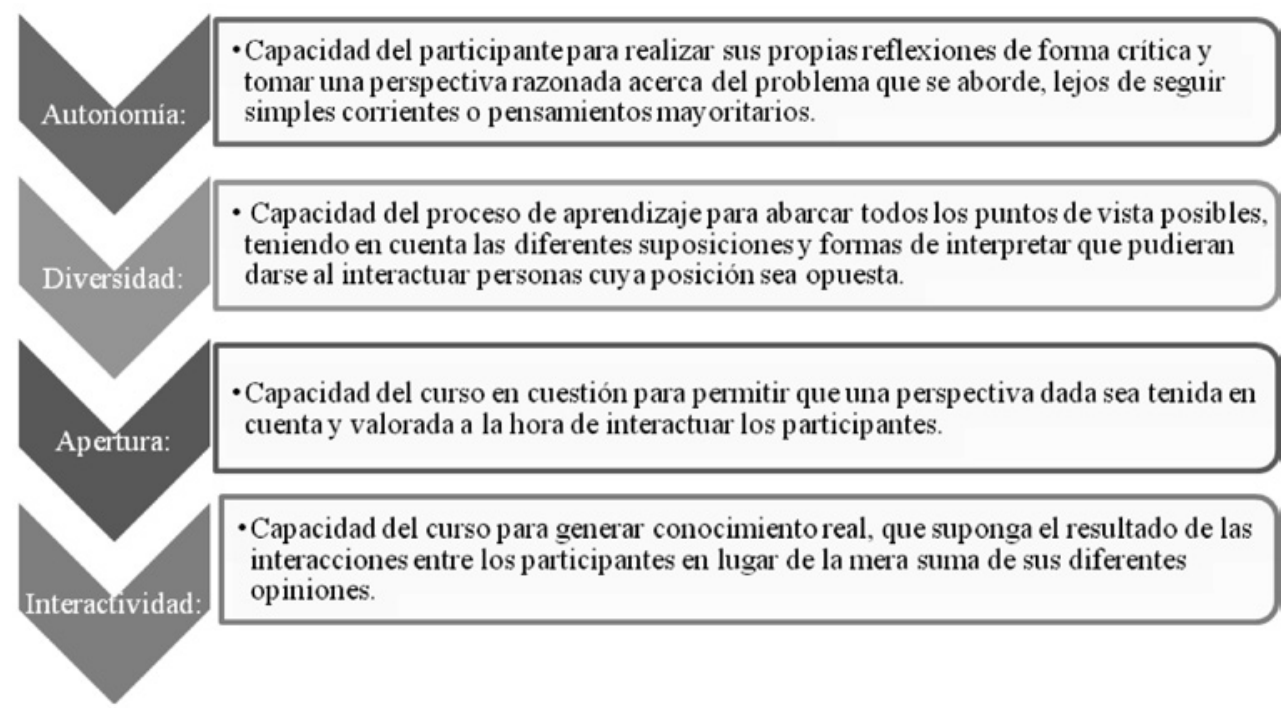

Figura 1. Principios de aprendizaje de los cMOOCs según Downes (2007)

Sin embargo, ciertos autores muestran algunas reticencias en relación a dichos principios, que resultan mucho más complejos de lo que pudieran aparentar, especialmente si se considera la experiencia personal y la propia identidad de cada estudiante (Mackness, Mak y Williams, 2010; Tschofen y Mackness, 2012). Por otra parte, la diversidad es entendida desde estos principios únicamente como el hecho de contemplar la realidad desde diferentes perspectivas, cuestión que se queda corta en relación a la amplitud que posee verdaderamente este término, y que incluye aspectos y cuestiones tan humanamente esenciales como las propias capacidades, conocimientos, competencias, creencias y/o cultura, entre otras.

En este sentido, desde los $c M O O C s$ se pueden plantear distintos trabajos que ofrezcan una respuesta adecuada a dicha diversidad. Krop y Carroll (2012) describen cuatro tipos diferentes de actividades dentro del aprendizaje conectivista que puede surgir en los cMOOCs y que se basa en las siguientes acciones principales: agregar, remezclar, crear y compartir (Figura 2). 


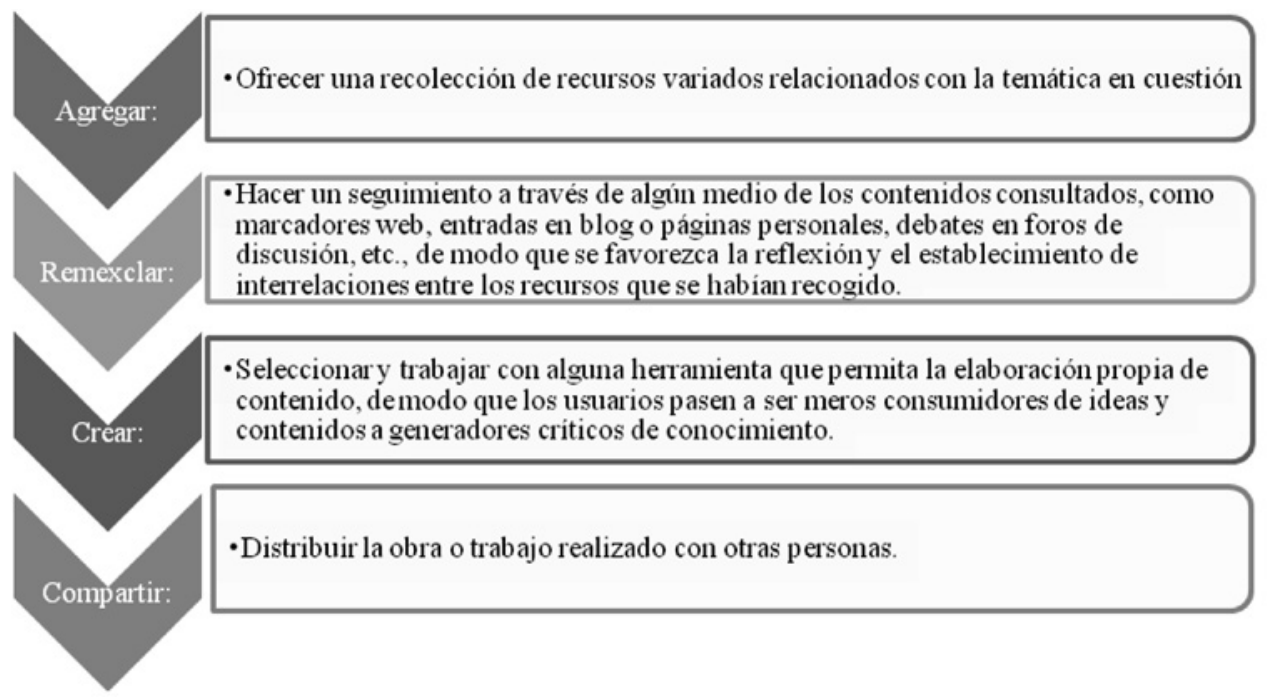

Figura 2. Acciones conectivistas propias de los $c M O O C s$

Ahora bien, sería necesario que todas estas acciones se adaptaran simultáneamente a las necesidades de cada estudiante, ofreciendo distintas posibilidades a modo de abanico de itinerarios formativos personales que permitan un aprendizaje global y único para cada usuario. Para ello, y especialmente en el momento de diseñar este tipo de cursos, el docente debe ser consciente de que deberá dejarse cierto espacio y posibilidades de acción durante el curso para poder efectuar posteriormente dicha adaptación, una vez se conozcan las necesidades de cada estudiante (que a su vez variarán a lo largo del mismo).

En cuanto a los comportamientos que cabe esperar por parte de los usuarios de este tipo de cursos, sería conveniente señalar que suelen mostrarse diferentes estilos (Kop, 2011): (a) el de mero consumidor de contenidos, que actúa de forma pasiva en la construcción de su propio aprendizaje, recibiendo unos conocimientos dados sobre los que no actúa ni interviene críticamente; y (b) el de productor de contenidos, que se comporta de modo activo a lo largo del curso, aprendiendo de y con sus compañeros, enseñándoles y compartiendo con ellos, elaborando sus propios conocimientos, etc. Así, sería este el tipo de comportamiento que debería fomentarse desde los $c M O O C s$ a fin de garantizar un verdadero intercambio de experiencias entre los usuarios que vele, a su vez, por el respeto y el conocimiento de la diversidad existente en el mismo.

Sin embargo, un comportamiento activo normalmente no nace en los usuarios per se, sino que debe favorecerse por medio del engagement. Según 
Milligan, Littlejohn y Margaryan (2013), algunos factores claves para que se produzca dicho engagement y que sin duda hay que considerar en el momento de diseñar un $c M O O C$ son la motivación, la confianza y la experiencia previa de los estudiantes. Pero tampoco debe olvidarse que otros factores que también involucran y logran el compromiso del alumnado en el proceso de aprendizaje conectivista propio de los $c M O O C$ s pasa por lo que Kop (2011) denomina "presencia social», tanto de los profesores como de los estudiantes, logrando la creación de una verdadera comunidad de aprendizaje y fomentando el sentido de pertenencia, aspectos que sin duda ayudan a construir confianza y estimular la participación activa de los usuarios. De esta forma se haría frente a la escasa participación de los usuarios en este tipo de cursos y se frenaría la considerable tasa de abandono que presentan. Además, siempre que se apueste por la creatividad, la autonomía, el aprendizaje social en red y la creación de contenidos propios por parte de sus alumnos (condiciones que persiguen todos los $c M O O C s$ ), se podrían alcanzar unos resultados personales de aprendizaje adecuados. Aunque para ello deberían proporcionarse, previamente y como ya se ha mencionado, medios y recursos adaptados a sus necesidades. Todo ello presupone un indicio de calidad en estos cursos que estaría lejos de conseguirse en otro tipo de $M O O C s$, especialmente los $x M O O C s$, que por su masividad complican enormemente la consecución de todos estos objetivos. En este sentido, parece razonable la propuesta de Valverde (2014) al criticar los xMOOCs por su escasa respuesta diferenciada a la diversidad, subrayando una nueva línea hacia el Diseño Universal del Aprendizaje (múltiples medios de representación, de acción-expresión y formas de implicación).

\section{ASEGURANDO LA CALIDAD DE LOS MOOCS}

El control de la calidad en los MOOCs parece ser una meta a alcanzar en la enseñanza a distancia. En la mayoría de los casos, especialmente en comparación con otros cursos en línea, se suele alegar que los MOOCs tienen una falta de estructura, apenas otorgan valor al rol del mediador, exigen un cierto nivel de alfabetización digital por parte de sus participantes e implican una experiencia educativa muy diferente a la tradicional, basándose fundamentalmente en el autoaprendizaje. Todo ello parece haber generado una seria preocupación sobre la inclusión y la igualdad de acceso a estos cursos, particularmente en los Estados en vías de desarrollo (Nyoni, 2013).

Antes de que surgieran los MOOCs ya había autores que proponían diferentes criterios para evaluar la calidad en los cursos virtuales existentes por el momento, como por ejemplo Santoveña (2005), pero sin contemplar aspectos relacionados con la atención a la diversidad. 
En consonancia y para garantizar la calidad de los MOOCs, Arias (2007) propone una serie de indicadores a tener en cuenta, sobre todo en consideración con la calidad pedagógica, que son: (a) la guía didáctica, (b) la metodología, (c) la organización de los contenidos, (d) la calidad de los contenidos, (e) los recursos didácticos, (f) la capacidad de motivación, (g) los elementos multimedia, (h) el estilo del lenguaje, (i) la discriminación y los valores y (j) la singularidad del usuario (p. 257). En cuanto a este último indicador, trata de medir la adecuación o no del sistema a las circunstancias personales y características particulares que presenta el usuario final. Por ello, la calidad en torno a la atención a la diversidad está presente, en mayor o menor medida, en la calidad de los MOOCs o, por lo menos y así consta, en la fundamentación teórica de la calidad de estos cursos.

\section{PROPUESTAS PARA ATENDER A LA DIVERSIDAD EN LOS MOOCS}

Como se ha visto en el apartado anterior, una de las principales condiciones para que un $M O O C$ conectivista sea de calidad es el grado de atención a la diversidad que ofrezca. Por tanto, sería conveniente destacar la idea de Méndez (2013), que afirma que los docentes han de ser previsores y tomar en consideración la diversidad de alumnado que van a recibir cuando trabajan a través de un $M O O C$, debido principalmente a sus características de apertura y masividad.

Por tanto, teniendo en cuenta las ideas mencionadas anteriormente y resaltando la baja atención prestada a la diversidad, a continuación se ofrecen una serie de pautas a introducir para mejorar en este aspecto.

La primera y más importante es garantizar que los MOOCs cumplan realmente y de manera eficaz una de sus premisas más extendidas y aceptadas: la masividad. Esta clase de cursos han de ser diseñados para acoger a todo el alumnado que esté interesado en participar y sin restringir sus destinatarios a un tipo de estudiantes totalmente homogéneo, ya que la riqueza en el intercambio de ideas y experiencias reside en la variedad.

Otro aspecto muy importante que se ha de tener en cuenta en cualquier curso, clase, asignatura... es conocer los conocimientos previos de los alumnos. Con ello se visualizan y ponen de manifiesto las dificultades y las capacidades que posee el grupo en cuestión para poder adaptar objetivos, contenidos, recursos, etc. a las necesidades grupales que presentan. Por ejemplo, si en el grupo hay algún alumno con discapacidad visual, se debe incluir todo el audio necesario para que no se pierda ninguna parte del curso. Además, y siguiendo con el mismo ejemplo, otra posibilidad para atender a este sector de alumnos es adaptar el $M O O C$ a un software como Jaws, el cual permite convertir el 
contenido de la pantalla en sonido. Una de las pautas que se debería poner también en práctica es incluir actividades de dificultad variable. De este modo, el curso queda totalmente abierto para poder atender a todos los públicos, independientemente de su cualificación, rama de sabiduría o conocimientos previos. Por ejemplo, se pueden incluir tareas de refuerzo para los alumnos con mayores dificultades y actividades de profundización para los expertos en la materia o para aquel que quiera aprender más sobre el contenido en cuestión.

Asimismo, un punto a favor de la diversidad que se puede considerar a la hora de elaborar un $M O O C$ en relación a las actividades que se proponen en su transcurso es introducir tareas con diferentes temáticas a elección de los alumnos. Con ello se pretende conseguir que los estudiantes se sientan atraídos y motivados durante el transcurso de su aprendizaje, evitando el abandono que se produce en muchos de los casos, a la vez que trabajan y se esfuerzan por aprender más sobre y con la materia. La motivación se puede conseguir al presentar una metodología activa que fomente la participación del alumnado en el proceso de enseñanza-aprendizaje, pero no es la única forma. También puede funcionar el resolver las dudas del alumnado de manera rápida, eficiente y profesional.

Además, siguiendo con la idea de que todos los alumnos son diferentes entre sí y parten de distintos niveles, sería conveniente adaptar, al igual que en el caso de las actividades, los exámenes en base a esas diferencias. Con ello estaríamos ante el hecho de proponer a los alumnos que se presenten a una modalidad de examen a elegir: uno de nivel de competencia medio y otro de nivel de competencia alto. En este sentido, no cabría olvidar las correspondientes adaptaciones y ayudas técnicas para aquellas personas con diversidad funcional.

Otra sencilla opción que pocas veces se contempla para atender a la diversidad es introducir en los vídeos utilizados subtítulos en lengua de signos para facilitar así su accesibilidad a todos los públicos.

Un aspecto importante a la hora de elaborar cursos masivos es, como se viene destacando, la confluencia variada de personas con características diversas. Por ello, sería conveniente adaptar los cursos a los diferentes estilos de aprendizaje. Así, lo ideal es que los alumnos pudieran tener a su alcance recursos como videotutoriales, mapas conceptuales, ejercicios prácticos, gráficas e ilustraciones, división por secuenciación de las tareas a realizar o los pasos a seguir para guiar el aprendizaje de forma más personalizada, etc. Para ello, una figura importante que habría de aparecer en estos cursos es la del profesor mediador o guía en el aprendizaje. Con él se solucionarían muchas de las dificultades que se encuentran los alumnos durante la visualización de vídeos, realización de tareas, participación en los foros, evaluación por pares, etc. 
En cuanto a esta última, la evaluación por pares es la estrategia más extendida y empleada en esta clase de cursos para la corrección de las tareas. En ella, los alumnos juegan un rol importante, ya que tienen la responsabilidad y el deber de revisar las tareas de sus compañeros. No obstante, en la mayoría de los casos, los alumnos se encuentran con un conflicto: en base a qué criterios evaluar. Por ello, una medida a tomar para atender a la diversidad del alumnado es que el docente revise las asignaciones para la corrección de las tareas de modo que el trabajo realizado concuerde con el nivel del alumnado que revisa el mismo, y ofrezca unos criterios de evaluación acordes con las características de cada alumno. De igual modo, resulta conveniente generar rúbricas que guíen y orienten a los estudiantes a la hora de evaluar los trabajos de sus compañeros en torno a una serie de criterios predefinidos a los que se otorga un determinado valor, de forma que los alumnos conocen de antemano qué aspectos deben valorar y qué resultados deben esperar por parte de estos. También es conveniente que el docente analice las calificaciones antes de aceptarlas y validarlas, por si se produjera algún error o injusticia en relación a los valores otorgados y/o las observaciones realizadas por el compañero revisor. De esta forma, se equilibran las puntuaciones, procurando que se adecúen al trabajo realizado por cada alumno y se contribuya a su aprendizaje incorporando comentarios adicionales por parte del docente, que ayuden a la subsanación de posibles errores conceptuales en la adquisición de conocimientos.

Los MOOCs se contemplan, en mayor medida, como una acción de apoyo a las universidades y, por tanto, a los estudios que estas ofrecen. No obstante, esta visión puede ir más allá y llegar a convertirse en estudios oficiales y contemplados como tal; por ejemplo: grado universitario. De este modo, con la explotación de los MOOCs se permitiría el acceso a estudios de este rango (universitarios) a personas que por determinadas razones (incompatibilidad horaria, movilidad reducida, enfermedades que exigen aislamiento, vivienda en zonas alejadas de la sede física, etc.) no puedan asistir de forma presencial, tanto a las clases como a la realización de los exámenes, prácticas y/o pruebas de nivel, y por tanto no tengan acceso a la obtención de dichos títulos.

Los MOOCs, como se desprende de todo lo dicho, ofrecen grandes oportunidades tanto para atender a la diversidad presente en ellos como para configurarse como un elemento de atención a la diversidad en sí mismos. Por tanto, y aunque las actuales medidas son escasas, no resultaría complejo implementar aquellas adaptaciones metodológicas que han sido expuestas en este apartado y garantizar con ello una mayor igualdad de oportunidades en estos cursos. 
A modo de resumen, se puede ejemplificar la propuesta metodológica de $M O O C$ mediante la secuenciación en tres fases esenciales (Tabla 1): diseño, desarrollo y evaluación. Las dos primeras etapas necesariamente siguen una sucesión, mientras que la evaluación puede realizarse paralelamente a las demás.

Tabla 1

Ejemplificación de propuesta metodológica y de secuenciación

\section{Diseño}

- Distintos niveles de profundización (p. e.: básico, medio y avanzado)

- Espacio virtual para intercambiar opiniones entre todos los participantes

- Guía de audio y manual sobre nociones básicas de conectividad, registro en la plataforma, descripción de estos cursos y accesibilidad y desarrollo de los mismos

- Adaptación a distintos lenguajes (p. e.: signos y subtítulos) e idiomas

- Metodología activa y participativa

- Diferentes contenidos sobre un mismo tema

- Combinación de distintos formatos en la presentación de la información

- Rúbricas de evaluación con criterios claramente definidos

\section{Desarrollo}

- Implantación y seguimiento del MOOC

- Revisión de las actividades y sus correspondientes evaluaciones

- Acción tutorial personalizada

\section{Evaluación}

- Incluir la atención a la diversidad como un criterio de calidad de los MOOCs

- Aplicar el Index for inclusion como una estrategia más de evaluación

\section{CONCLUSIONES}

La vida de los MOOCs es todavía corta, por lo que aún se necesitan perfilar algunas incongruencias internas que presentan, de índole esencial y compleja, como por ejemplo la atención a la diversidad. Alguna tipología 
específica de estos cursos, como los $c M O O C$ s, está intentando crecer en este aspecto fomentando un aprendizaje conectivista. Esto significa que cada vez se producen con más frecuencia redes de aprendizaje entre los alumnos, en las que pasan de desempeñar un papel de meros consumidores de información a ser productores y transmisores de contenidos; es decir, rompiendo con las estructuras y los roles tradicionales. No obstante, es largo el camino que queda para conseguir una verdadera atención a la diversidad en ellos.

Es una evidencia que, aunque generalmente los MOOCs muestran sesgos hacia la diversidad de su alumnado, es posible una mejora en este sentido. Tal diversidad puede verse favorecida por la masividad y apertura característica de estos cursos, lo que implica un gran abanico de posibilidades en torno a los conocimientos previos, intereses, competencias tecnológicas (Méndez, 2013), estilos de aprendizaje (Grünewald y otros, 2013), lenguas y culturas, perfiles (Kop, 2011), etc. En esta línea, cabe romper la tendencia actual de considerar a estos principios metodológicos de los MOOCs como sinónimos de homogeneidad, para así apreciar la heterogeneidad del alumnado y aprender con, para y de ella, lo que es un principio e indicador de calidad (Arias, 2007; Downes, 2007).

No obstante, el aspecto masivo también conlleva elementos negativos, propiciando, por ejemplo, situaciones que desembocan en una gran tasa de abandono. Pese a que a partir del análisis masivo de datos cuantitativos se pueden crear perfiles para personalizar el aprendizaje (Aguaded, 2013; Calderón y otros, en prensa; Kock, 2009), este hecho hace que se trate a los alumnos como números y no como personas y, por tanto, se desconsideren otras variables de aprendizaje que tienen lugar fuera de estos cursos. Es decir, se puede personalizar el aprendizaje, pero de forma descontextualizada, lo que conlleva que se olviden las diferencias humanamente esenciales (Mackness y otros, 2010; Tschofen y Mackness, 2012).

Para apreciar a los alumnos como personas y valorar sus características, es fundamental la gestión de las redes de aprendizaje por parte del docente. Una de sus principales características es que fomentan el sentido de pertenencia (Kop, 2011), facilitando así la inclusión del alumnado dentro del grupo y, por tanto, postulando la atención a la diversidad como un elemento enriquecedor para todos los participantes.

En cuanto a la característica de apertura que presentan los MOOCs, la igualdad de acceso (Nyoni, 2013) se ve condicionada fundamentalmente por las competencias tecnológicas que presentan los alumnos y las condiciones informáticas del entorno, es decir, no basta con tener conocimientos tecnológicos, sino que hay que contar con recursos y acceso a estos, lo cual en muchos casos no es posible. 
Por tanto, y con el propósito de mejorar la atención a la diversidad en los MOOCs, es conveniente introducir mejoras metodológicas como las recogidas en el presente estudio. No obstante, sería oportuno seguir investigando sobre esta cuestión a fin de que estos cursos puedan atender verdaderamente a la diversidad. A pesar de seguir verdaderamente unos principios orientados hacia la inclusión de todo el alumnado, haciendo frente a esta propuesta, requiere una gran cantidad de recursos que se consideran necesarios para implementarla, especialmente los referentes a recursos personales (tutores virtuales). 


\section{REFERENCIAS BIBLIOGRÁFICAS}

Aguaded, J. I. (2013). La revolución MOOCs, ¿una nueva educación desde el paradigma tecnológico? Comunicar, 41, 7-8. Recuperado de http://dx.doi. org/10.3916/C41-2013-a1

Calderón, J. J., Ezeiza, A. y Jimeno, M. (en prensa). La falsa disrupción de los MOOC: la invasión de un modelo obsoleto. En G. J. Palacios (Eds.), VI Congreso Internacional de Educación Abierta y Tecnología. Bilbao: Universidad del País Vasco.

Downes, S. (2007). An Introduction to Connective Knowledge. En T. Hug (Ed.), Media, Knowledge \& Education. Exploring new Spaces, Relations and Dynamics in Digital Media Ecologies (pp. 77-102). Inssbruck: Inssbruck University Press.

Griesbaum, J. (2014). Students as Teachers in MOOCs? The Double Gain of MOOCs as an in-Class Teaching Method Experiences from a StudentMade MOOC «Online Data Privacy». International Journal of Information and Education Technology, 4 (1).

Grünewald, F., Meinel, C., Totschnig, M. \& Willems, C. (2013). Designing MOOCs for the support of multiple learning styles. En D. Hernández-Leo, T. Ley, R. Klamma y A. Harrer (Eds.), Scaling up learning for sustained impact (pp. 371-382). Berlín: Springer.

Karsenti, T. (2013). The MOOC: what the research says. International Journal of Technologies in Higher Education, 10(2), 23-37.

Köck, M. (2009). Towards intelligent adaptive e-learning systems. Machine learning for learner activity classification. En D. Hauger, M. Köck y A. Nauerz (Eds.), Proceedings of the 17th Workshop on Adaptivity and User Mo- deling in Interactive Systems (pp. 2631). Linz: Johannes-Kepler-University.

Kop, R. (2011). The challenges to connectivist learning on open online networks: Learning experiences during a massive open online course. International Review of Research in Open and Distance, 12 (3), 19-38. Recuperado de https://goo.gl/quZ5ZA

Kop, R. \& Carroll, F. (2012). Cloud computing and creativity: Learning on a massive open online course. European Journal of Open, Distance and E-learning, 12. Recuperado de https://goo.gl/v9ndx4

Kop, R. y Hill, A. (2008). Connectivism: Learning theory of the future or vestige of the past? International Review of Research in Open and Distance Learning, 9 (3). Recuperado de https://goo. $\mathrm{gl} / \mathrm{I} 8 \mathrm{bVa}$

Levy, D. (2011). Lessons learned from participating in a connectivist massive online open course (MOOC). En Y. Eshet-Alkalai, A. Caspi, S. Eden, N. Geri y Y. Yair (Eds.), Learning in the Technological Era (pp. 31-36). Raanana: Open University of Israel.

Liyanagunawardena, T. R., Adams, A. A. y Williams, S. A. (2013). MOOCs: a systematic study of the published literature 2008-2012. International Review of Research in Open and Distance Learning, 14 (3), 202-227. Recuperado de https://goo.gl/VMOqt

Mackness, J., Mak, S. F. J. \& Williams, R. (2010). The ideals and reality of participating in a MOOC. En L. Dirckinck-Holmfeld, V. Hodgson, C. Jones, M. de Laat, D. McConnell y T. Ryberg (Eds.), Proceedings of the 7th International Conference on Networked Learning (pp. 266-274). Bailrigg: Lancaster University. 
Mackness, J., Waite, M., Roberts, G. y Lovegrove, E. (2013). Learning in a small, task-oriented, connectivist MOOC: Pedagogical issues and implications for higher education. International Review of Research in Open and Distance Learning, 14 (4), 140-159. Recuperado de https://goo.gl/8HqMa3

Martín-Monge, E., Bárcena, E. y Read, T. (2014). La interacción entre compañeros y el feedback lingüístico en los COMA de lenguas extranjeras. Profesorado. Revista de Currículum y Formación del Profesorado, 18 (1), 167-183.

Méndez, C. M. (2013). Diseño e implementación de cursos abiertos masivos en línea: expectativas y consideraciones prácticas. Revista de Educación a Distancia, 13 (39). Recuperado de https:/goo.gl/7H0P0X

Milligan, C., Littlejohn, A. y Margaryan, A. (2013). Patterns of Engagement in Connectivist MOOCs. Journal of Online Learning and Teaching, 9 (2). Recuperado de https://goo.gl/7yUXz4

Nyoni, J. (2013). The Viral Nature of Massive Open Online Courses (MOOCs) in Open and Distance Learning: Discourses of Quality, Mediation and Control. Mediterranean Journal of Social Sciences, 4 (3), 665-672. Recuperado de http://DOI: 10.5901/mjss.2013. v4n3p665.

Odom, L. (2013). A SWOT Analysis of the potential impact of MOOCs. En
J. Herrington, A. Couros \& V. Irvine (Eds.), Proceedings of Word Conference on Educational Multimedia, Hypermedia and Telecommunications (pp. 611-621). Tampere: Association for the Advancement of Computing in Education.

Santoveña, S. (2005). Criterios de calidad para la evaluación de cursos virtuales.Etic@net, 2 (4),18-36. Recuperado de http://goo.gl/lwFZ5

Siemens, G. (2005). Connectivism: A Learning Theory for the Digital Age. International Journal of Instructional Technology and Distance Learning, 2 (1). Recuperado de https://goo. $\mathrm{gl} / 7 \mathrm{PgUQ}$

Torres-Díaz, J. C., Infante, A. y Valdiviezo, P. (2014). Los MOOC y la masificación personalizada. Profesorado. Revista de Currículum y Formación del Profesorado, 18 (1), 63-72.

Tschofen, C. \& Mackness, J. (2012). Connectivism and dimensions of individual experience. International Review of Research in Open and Distance Learning, 13 (1), 124-143. Recuperado de https://goo.gl/53NZt

Valverde, J. (2014). Una visión crítica desde las Ciencias de la Educación. Profesorado. Revista de Currículum y Formación del Profesorado, 18(1), 93-111.

Watters, A. (2013). Got MOOC? School Library Journal, 59 (4), 29-31. 


\section{PERFIL ACADÉMICO Y PROFESIONAL DE LOS AUTORES}

Alba García Barrera. Doctora en Educación por la Universidad Autónoma de Madrid. Profesora Adjunta de la Facultad de Ciencias de la Salud y Educación de la Universidad a Distancia de Madrid, donde dirige el Máster en Dirección y Gestión de Centros Educativos. Miembro del Grupo de Investigación «Enseñanza, Aprendizaje y Nuevas Tecnologías» (EA-TIC). Sus principales líneas de investigación giran en torno a la atención a la diversidad, la innovación didáctica y la tecnología educativa.

Patricia Gómez Hernández. Doctoranda en Educación en la Universidad de Alcalá. Contratada predoctoral (FPU-UAH) en el Departamento de Ciencias de la Educación (área de Psicología Evolutiva y de la Educación). Sus principales líneas de investigación giran en torno a la innovación educativa mediada por TIC.

Carlos Monge López. Doctorando en Educación en la Universidad de Alcalá. Contratado pre-doctoral (FPU-UAH) en el Departamento de Ciencias de la Educación (área de Didáctica y Organización Escolar). Miembro del grupo de investigación «Inclusión y Mejora Educativa: Convivencia y Aprendizaje Cooperativo» (UAH). Sus principales líneas de investigación giran en torno a la inclusión e innovación educativa.

Dirección de los autores: Alba García Barrera

Universidad a Distancia de Madrid

Facultad de Ciencias de la Salud y Educación

Carretera de A Coruña, km 38,5

Vía de servicio, n. ${ }^{\circ} 15$

28400 Collado Villalba - Madrid

E-mail: alba.garcia@udima.es

Patricia Gómez Hernández

Universidad de Alcalá

Aulario María de Guzmán

C/ San Cirilo, s/n

28801 Alcalá de Henares - Madrid

E-mail: Patricia.gomezh@edu.uah.es

Carlos Monge López

Universidad de Alcalá

Facultad de Educación

C/ Madrid, 1, despacho 321

19001 Guadalajara

E-mail: carlos.monge@uah.es

Fecha Recepción del Artículo: 28. Septiembre. 2014

Fecha modificación del Artículo: 29. Enero. 2015

Fecha Aceptación del Artículo: 08. Febrero. 2015

Fecha Revisión para Publicación: 08. Marzo. 2017 
